\title{
Methamphetamine: Forbidable but Still a Forensic Issue
}

\author{
Mohammed Nasimul Islam* and Jesmine Khan
}

Faculty of Medicine, University Teknologi MARA, Selangor, Malaysia

\begin{abstract}
Methamphetamine (MA) abuse is one of the challenging problems in Forensic Pathology. It has adverse effects on heart, intestine and male reproductive system. In 1990's it was identified as an alarming problems among the adolescents. Thus the agent was included as a controlled substance. This has created two main problems. In one hand, the scientists engaged in research in this field have failed to carry forward their research projects due to scarcity of the substance. On the other hand, plenty of this substance is illegally manufactured and available in the black market especially at the border area. The article explains the current situation of MA abuse and health conditions after withdrawal of the agent.
\end{abstract}

Keywords: Methamphetamine; Cardiomyopathy; Villas height; Histology; Male reproductive system; Withdrawal effects

Methamphetamine $\mathrm{HCl}(\mathrm{MA})$ is one of the common drugs that by drug abusers use. It is available from north to south and east to west under various names eg: crystal meth, speed, hot ice, tina, glass, sabu etc [1]. As MA is associated with a high potential for misuse, the drug is regulated under the Controlled Substances Act and is listed under Schedule II. Each and every country in the planet has been trying to eradicate this drug from their black market. But in course of time, it has become popular among the drug abusers and thusits demandhas increased. Statistics show that half of teens have tried some sort of illicit drug by the time they finish high school, and over 12 million adolescents have tried MA in their life for at least once [2]. In order to clarify its adverse effect of this agent, among others the authors had carried out an extensive search on heart, intestines and the male reproductive system. Interestingly, there were many obstacles to get MA even for research purposes. In 1990s it could easily be obtained from the pharmaceutical companies. Later, it became included in schedule II group and declared formidable item to export. This decision of the drug administration did not help to stop the use of this drug, rather the researchers were forced to switchover their thoughts in different directions. Although, Illicit MA of $95 \%$ purity is available in the black market, but that cannot be used in research on ethical grounds.

MA is a non-catecholamine sympathomimetic drug which has been increasingly used by drug abusers for elevating their mood, libido and decreasing appetite and fatigue [3-5]. Although, in lower dosages, MA can increase alertness, concentration, and energy in fatigued individuals. Chronic abuse may lead to post-withdrawal syndrome, a result of MA-induced neurotoxicity to dopaminergic neurons. Postwithdrawal syndrome can persist beyond the withdrawal period for months, and sometimes up to a year [6]. In addition to psychological harm, physical harm -primarily consisting of cardiovascular damage may occur with chronic use or acute overdose [7].

In many countries of the world, MA has been medically used. In USA, this drug is approved by the Food and Drug Administration (FDA) in treating ADHD and exogenous obesity in both adults and children [8].

A number of sympathomimetic amines are capable of myocardial damage but cardiotoxic action of MA has been of particular interest since standardized dosage consistently produces myocardial lesions. Sudden death from cardiac causes in MA users is one of the challenging problems in medical science, especially in forensic pathology $[6,9,10]$.
Recent study also confirmed its adverse effects on intestinal villi $[11,12]$. It has also effect on male reproductive system [7]. To diagnose the pathological events of the heart and intestine, routine macroscopic and microscopic examinations are very reliable, but the interpretation of these studies is not always easy. Besides this, the combination of several methods gives the best result. The author therefore carried out an extensive search through histological, immunohistochemical and electron microscopic examinations on the same tissue to see if more cases of a probable pathology could be diagnosed, either as positive or negative, with greater certainty. It is considered as routine determinations in most laboratories of forensic medicine for the diagnosis of histo-pathology [8].

Light microscopic changes found in the MA treated rat myocardium included atrophy, hypertrophy, patchy cellular infiltration, eosinophilic degeneration and disarray, edema, myolysis, fibrosis and the appearance of vacuoles. Ultra structurally, nuclei and normal mitochondria of various shape, dilated $\mathrm{T}$ tubules and sarcoplasmic reticulum, accumulation of glycogen granules and fat droplets, intra and extracellular edema and intra-myocytic vacuoles. In MA treated small intestine, villus heights were decreased and goblet cells were increased dramatically as compared to normal and placebo treated samples [9]. In male reproductive system it was evident that seminiferous tubular diameter and seminiferous epithelial height was significantly reduced [7].

MA has a high potential for abuse and should be tried only in weight reduction programs where alternative therapy has been ineffective. Administration of MA for prolonged periods may lead to drug dependence. This drug should be prescribed or dispensed sparingly. Misuse may cause sudden death and serious cardiovascular adverse events [11].

*Corresponding author: Mohammed Nasimul Islam, Faculty of Medicine, University Teknologi MARA (UiTM), Jalan Hospital, 47000 Sungai Buloh, Selangor, Malaysia, Tel: +603-5521-1284; E-mail: nasimul@salam.uitm.edu.my

Received November 25, 2013; Accepted November 27, 2013; Published November 29, 2013

Citation: Islam MN, Khan J (2013) Methamphetamine: Forbidable but Still a Forensic Issue. J Forensic Res 4: e114. doi:10.4172/2157-7145.1000e114

Copyright: (C) 2013 Islam MN, et al. This is an open-access article distributed under the terms of the Creative Commons Attribution License, which permits unrestricted use, distribution, and reproduction in any medium, provided the original author and source are credited. 
In spite of increasing adoption of MA by drug abusers, it rarely represents the end stage of the spectrum of drug addiction, as the addicts soon alter their choice to narcotics or other drugs [3]. Thus the findings those were claimed by the scientists were produced either by MA alone or by the combination of amphetamine or other drugs those have taken by abusers in course of time. Nevertheless, the information available to date suggests that dosage based on intake of this agent by the abusers although helpful in obesity but in greater aspects endangers life of its users. Histopathological findings after withdrawal of $\mathrm{MA} \mathrm{HCl}$ were not disappointing. Almost all the MA induced changes in the heart were found to be reversed by its withdrawal except the fibrotic one. Apart from the myocardial changes, small intestinal villus height and goblet cells and pathology of male reproductive system has returned back to normal in course of time. Therefore, there is a possibility that MA abusers return back to normal life if they withdraw from the intake of this agent.

\section{References}

1. Derlet RW, Heischober B (1990) Methamphetamine-Stimulant of the 1990s? West J Med 153: 625-628.

2. http://casapalmera.com/nicknames-street-names-and-slang-formethamphetamine/
3. Hong R, Matsuyama E, Nur K (1991) Cardiomyopathy associated with the smoking of crystal methamphetamine. JAMA 265: 1152-1154.

4. Kobayashi M (1986) Epidemiology of Drug dependence. Clin Neur Sci 4: 774 778.

5. Swenson JR, Dimsdale JE, Rockwell E, Carroll W, Hansbrough J (1991) Drug and alcohol abuse in patients with acute burn injuries. Psychosomatics 32: 287 293.

6. Cruickshank CC, Dyer KR (2009) A review of the clinical pharmacology of methamphetamine. Addiction 104: 1085-1099.

7. Yusof MFB, Khan J, Islam MN (2013) Withdrawal Effects of Long Term Administration of Methamphetamine on the Reproductive System of Male Rats. International Medical Journal 20: $56-58$

8. Islam MN, Kuroki H, Hongcheng B, Ogura Y, Kawaguchi N, et al. (1995) Cardiac lesions and their reversibility after long term administration of methamphetamine. Forensic Sci Int 75: 29-43.

9. Khan J, Islam MN (2008) Effect of Methamphetamine on the Small Intestinal Morphology of Rats. International Medical Journal 15: 385-388.

10. Matoba R, Onishi S, Shikata I (1985) Cardiac Lesions in case of sudden death in methamphetamine abusers. Heart and Vessels 1: 298-300.

11. Desoxyn (Methamphetamine Hydrochloride) Drug Information: User Reviews, Side Effects, Drug Interactions and Dosage at. Rxlist.

12. Matoba R, Shikata I, Iwai K, Onishi S, Fujitani N, et al. (1989) An epidemiologic and histopathological study of sudden cardiac death in Osaka Medical Examiner's Office. Jpn Circ J 53: 1581-1588. 